\title{
High-spatial-resolution deep tissue imaging with spectral-domain optical coherence microscopy in the $1700-\mathrm{nm}$ spectral band
}

Masahito Yamanaka

Naoki Hayakawa

Norihiko Nishizawa 


\section{High-spatial-resolution deep tissue imaging with spectral-domain optical coherence microscopy in the 1700-nm spectral band}

\author{
Masahito Yamanaka, ${ }^{*}$ Naoki Hayakawa, and \\ Norihiko Nishizawa \\ Nagoya University, Department of Electronics, Nagoya, Aichi, Japan
}

\begin{abstract}
We present three-dimensional (3-D) highresolution spectral-domain optical coherence microscopy (SD-OCM) by using a supercontinuum (SC) fiber laser source with 300-nm spectral bandwidth (full-width at halfmaximum) in the 1700-nm spectral band. By using lowcoherence interferometry with SC light and a confocal detection scheme, we realized lateral and axial resolutions of 3.4 and $3.8 \mu \mathrm{m}$ in tissue $(n=1.38)$, respectively. This is, to the best of our knowledge, the highest 3-D spatial resolution reported among those of Fourier-domain optical coherence imaging techniques in the 1700-nm spectral band. In our SD-OCM, to enhance the imaging depth, a full-range method was implemented, which suppressed the formation of a coherent ghost image and allowed us to set the zero-delay position inside the samples. We demonstrated the 3-D high-resolution imaging capability of $1700-\mathrm{nm}$ SD-OCM through the measurement of an interference signal from a mirror surface and imaging of a single 200-nm polystyrene bead and a pig thyroid gland. Deep tissue imaging at a depth of up to $1.8 \mathrm{~mm}$ was also demonstrated. This is the first demonstration of 3-D high-resolution SD-OCM in the 1700-nm spectral band. (c) The Authors. Published by SPIE under a Creative Commons Attribution 4.0 Unported License. Distribution or reproduction of this work in whole or in part requires full attribution of the original publication, including its DOI. [DOI: 10.1117/1.JBO.24.7.070502]
\end{abstract}

Keywords: spectral-domain optical coherence microscopy; 1700-nm spectral band; three-dimensional high-resolution; deep tissue imaging.

Paper 190131LR received Apr. 20, 2019; accepted for publication Jul. 10, 2019; published online Jul. 30, 2019.

\section{Introduction}

Optical coherence microscopy (OCM) is an optical coherence imaging technique based on optical coherence tomography (OCT) combined with confocal microscopy using a highnumerical-aperture (NA) objective lens, which allows us to perform three-dimensional (3-D) high-resolution, label-free imaging of biological specimens without any physical or chemical pretreatment, such as staining or sectioning. ${ }^{1}$ So far,

*Address all correspondence to Masahito Yamanaka, E-mail: yamanaka .masahito@h.mbox.nagoya-u.ac.jp
OCM techniques in the 800 - to 1300 -nm spectral band, which enable noninvasive, label-free visualization of biological structures in 3D with high resolution, have been widely employed for biomedical studies. ${ }^{2-5}$ In addition, multimodal imaging techniques to integrate OCM with other optical imaging modalities, such as fluorescence microscopy, photoacoustic microscopy, and so on, have also been reported to provide complementary features of biological specimens, allowing more detailed biological understanding. ${ }^{6,7}$

In the development of OCM and OCT systems currently underway, one of the important goals is to realize a large imaging depth in tissue imaging. In tissue imaging with OCM/OCT using near-infrared (NIR) light, the imaging depth of OCM/ OCT is limited by light attenuation due to multiple light scattering and light absorption events by water inside the samples. To achieve greater imaging depth in the observation of turbid scattering tissue, light sources in the 1300-nm spectral band are commonly used, because the light scattering is reduced at $1300 \mathrm{~nm}$ compared to the conventional NIR band of 650 to $900 \mathrm{~nm}$. For example, an imaging depth of $2.3 \mathrm{~mm}$ in the imaging of living mouse brains was demonstrated by using 1300-nm swept-source (SS) OCT. ${ }^{8}$ A 1300-nm spectral-domain (SD) OCM system was also reported, and this system realized 3-D high-resolution imaging of living rat brain tissues with an imaging depth of $\sim 1.3 \mathrm{~mm} .^{5}$ Although the light absorption by water becomes higher in the $1700-n m$ wavelength region, several groups, including ours, have recently reported that use of the 1700-nm spectral band allows imaging at an imaging depth greater than that in the 1300-nm spectral band, due to the lower light attenuation, which arises from the reduced scattering coefficient and the existence of a local minimum of the water absorption coefficient in the $1700-\mathrm{nm}$ spectral band. ${ }^{9-19}$ In recently reported 1700-nm SS-OCT, an imaging depth of $2.6 \mathrm{~mm}$ was realized in imaging of living mouse brains. ${ }^{20}$ In our group, we have been developing high-axial-resolution 1700-nm OCT by using a supercontinuum (SC) fiber laser source. Based on our OCT system, we also realized 3-D high-resolution timedomain (TD) OCM in the 1700-nm spectral band \{ the center wavelength is $1730 \mathrm{~nm}$ and the spectral bandwidth is $380 \mathrm{~nm}$ [full-width at half-maximum (FWHM)] , which enabled lateral and axial resolutions of 1.3 and $2.8 \mu \mathrm{m}$ (in tissue). ${ }^{21}$ In addition, a comparison of the imaging depths of 1700 and $1300 \mathrm{~nm}$ TDOCM under the same sensitivity conditions showed that the 1700 -nm spectral band is a promising choice for deep-tissue imaging with OCM. ${ }^{21}$ However, because the optical pass length of the reference beam was scanned by a mechanical scanner, the image acquisition time of the high-resolution 1700-nm TDOCM was not sufficient for biological studies (image acquisition time: $\sim 40$ min for a single en-face image with $512 \times$ 512 pixels). In addition, the signal detection sensitivity was also limited to around $93 \mathrm{~dB}$, due to the use of such a broad spectral bandwidth in a TD detection system. ${ }^{21,22}$

In this study, to improve the image acquisition speed and signal detection sensitivity of high-resolution 1700-nm OCM, we developed 3-D high-resolution $1700 \mathrm{~nm}$ spectral-domain optical coherence microscopy (SD-OCM) by using an SC fiber laser source with a 300-nm spectral bandwidth (FWHM) in the 1700-nm spectral band. This SD-OCM was based on our TD$\mathrm{OCM}^{21}$ and a full-range high-axial-resolution SD-OCT system operating in the 1700 -nm spectral band. ${ }^{16}$ The full-range method was employed to realize both high axial resolution and large imaging depth. In the 1700-nm spectral band, to achieve high 
axial resolution, a spectral bandwidth of more than several hundred nanometers is required. The use of such a broad bandwidth usually sacrifices the spectral resolution due to the limited number of pixels of the line-scan camera, resulting in a large sensitivity roll-off. Because the formation of a coherent ghost image forces us to set the zero-delay position outside the samples to avoid the overlapping of a complex conjugate image with an OCM/OCT image, the large sensitivity roll-off limits the imaging depth of high-axial-resolution 1700-nm SD OCM/OCT. The full-range method allows us to set the zero-delay position, where the highest sensitivity is achieved, inside the samples by suppressing the formation of a coherent ghost image, leading to OCM/OCT imaging with a large imaging depth and high axial resolution. In this study, we confirmed that the developed 1700-nm SD-OCM offers lateral and axial resolutions of 3.4 and $3.8 \mu \mathrm{m}$ in tissue $(n=1.38)$, respectively, with a sensitivity of $100 \mathrm{~dB}$ and an image acquisition time of $6 \mathrm{~s}$ for a single en-face image with $512 \times 512$ pixels, and then showed the merits of extending $1700 \mathrm{~nm}$ OCT to OCM by comparing en-face tissue images obtained by $1700 \mathrm{~nm}$ OCT and OCM with the same axial resolution. We also demonstrated deep tissue imaging at a depth of up to $1.8 \mathrm{~mm}$ by observing a pig thyroid gland. To the best of our knowledge, the 3-D spatial resolution of this $1700-\mathrm{nm}$ SD-OCM is the highest reported among Fourierdomain optical coherence imaging techniques in this wavelength region. Although the achievable axial resolution of the 1700-nm SD-OCM becomes slightly lower than that of the 1700-nm TD-OCM, due to the narrower spectral bandwidth caused by the spectrometer-based detection system (the spectral bandwidth of the 1700-nm TD- and SD-OCM: 380 and $300 \mathrm{~nm}$ ), the developed SD-OCM system provides the improved image acquisition speed and sensitivity, which makes high-resolution 1700-nm OCM more useful for biological studies.

\section{High-Spatial-Resolution Spectral-Domain Optical Coherence Microscopy System in the 1700-nm Spectral Band}

Figure 1(a) shows the experimental setup for 1700-nm SDOCM using an SC fiber laser source and a custom-built broadband spectrometer equipped with a line scan InGaAs camera (Goodrich SU1024LDH-2.2RT-0250 = LC), which are basically the same as those described in Ref. 16. In this SD-OCM system, the SC light was divided into reference and sample beams through the Michelson interferometer using a broadband fiber coupler. The sample arm was composed of optics for laser scanning microscopy with a two-axis galvanometer scanner. The SC light was focused on samples with an objective lens having an NA of 0.45 (LCPLN20XIR, Olympus). This objective lens had a transmittance of more than $60 \%$ in the $1700-\mathrm{nm}$ spectral band. In the sample arm, the core of the fiber worked as a confocal gate. To perform full-range OCM imaging, we employed a phase modulation method. The details of this full-range method are described in Ref. 16. The light scattered and reflected back from the sample and reference arms was made to interfere in the fiber coupler and then detected with the line scan InGaAs camera. The line rate of this camera was $47 \mathrm{kHz}$, and a 150grooves/mm blazed diffraction grating was used for the spectrometer (015-200, Shimadzu). Compensation of the chromatic dispersion and polarization mismatching between the sample and reference arms was performed with optical glasses placed in the reference arm and polarization controllers.

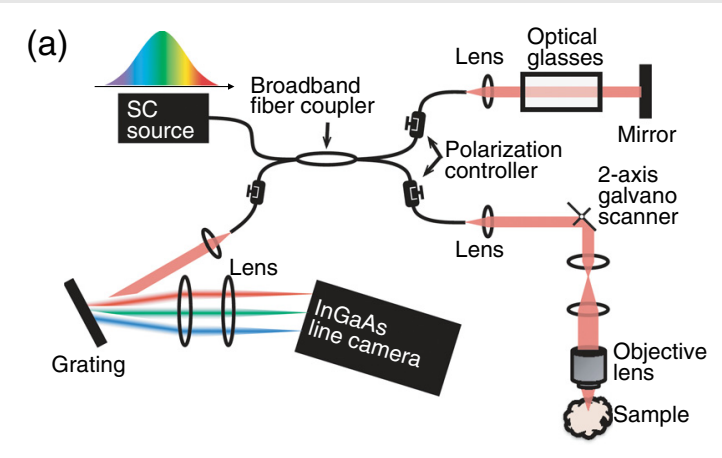

(b)

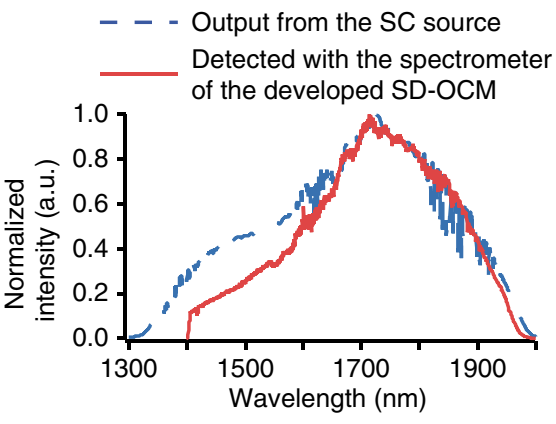

Fig. 1 (a) Experimental setup for 3-D high-resolution 1700-nm SDOCM using the SC fiber laser source in the 1700-nm spectral band. (b) Optical spectra of the output light from the SC source (blue dotted line) and light detected with the spectrometer equipped with the line scan InGaAs camera (red line).

Our SC fiber laser source was composed of an ultrashort erbium-doped fiber laser, erbium-doped fiber amplifier, polarization maintaining fiber, and highly nonlinear fiber with normal dispersion properties. ${ }^{11,12,16,21}$ The spectral bandwidth of the output light from the SC source was $360 \mathrm{~nm}$ (FWHM), and the output power was $30 \mathrm{~mW}$. Although the spectral bandwidth of the detected interference signal was slightly decreased due to the limited bandwidth of the spectrometer and fiber coupler, the spectral bandwidth still reached $300 \mathrm{~nm}$ FWHM [Fig. 1(b)]. The theoretical limit of the axial resolution obtained with this experimental condition is $3.2 \mu \mathrm{m}$ in tissue $(n=1.38)$.

To evaluate the sensitivity and axial resolution of the developed SD-OCM, we measured reflected light from a single mirror surface. Figures 2(a) and 2(b) show log and linear plots of the measured OCM signal, respectively. In this measurement, to avoid saturation of the line scan camera, a neutral density (ND) filter was inserted into the sample arm. The laser power on the sample was $6 \mathrm{~mW}$ and the round-trip attenuation ratio with this ND filter was $39 \mathrm{~dB}$. From Figs. 2(a) and 2(b), we confirmed that the sensitivity was $100 \mathrm{~dB}$ and the axial resolution was $5.2 \mu \mathrm{m}$ in air, which corresponds to $3.8 \mu \mathrm{m}$ in tissue $(n=1.38)$. Although the spectral bandwidth in tissue imaging is reduced by strong light absorption by water at 1.45 and $1.9 \mu \mathrm{m}$ wavelength regions, our previous study on the axial resolution in deep tissue imaging with 1700-nm OCT using the broadband light source showed that it is still possible to achieve the axial resolution of $4.3 \mu \mathrm{m}$ in tissue. ${ }^{18}$

The lateral resolution was confirmed by observing a single 200-nm polystyrene bead embedded in gelatin. As shown in Fig. 2(c), the FWHM of the intensity profile of the single bead was $3.4 \mu \mathrm{m}$. Because the bead diameter was much smaller than the diffraction limited resolution in the lateral direction, this intensity profile almost corresponds to the point spread function. 
(a)

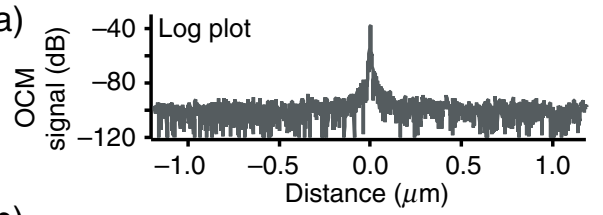

(b)

(c)

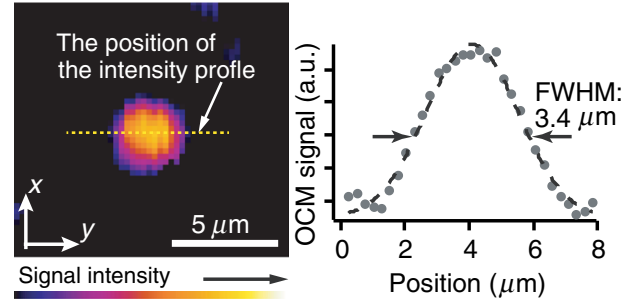

Fig. 2 (a) Log and (b) linear plots of the measured interference signal from a single mirror surface and (c) OCM image of a single 200-nm polystyrene bead embedded in gelatin and intensity profile (yellow dotted line) indicated by the white arrow.

From this result, we confirmed that the lateral resolution of our SD-OCM system was $3.4 \mu \mathrm{m}$.

\section{Tissue Imaging with the Developed SD-OCM}

To demonstrate the deep tissue imaging capability of the developed SD-OCM, we observed a normal adult pig thyroid gland. For this experiment, we purchased a pig thyroid gland from a company, and the organ was delivered in cold storage $\left(4^{\circ} \mathrm{C}\right)$ vation, the sample was immersed in phosphate-buffered saline solution. Figure 3 shows the en-face OCM images of a normal pig thyroid gland at depths of $0.3,0.6,0.9,1.2,1.5$, and $1.8 \mathrm{~mm}$ from the sample surface. Because the dry objective lens (LCPLN20XIR, Olympus) was used for this experiment, the optical pass length in the sample arm increased with the increase of the imaging depth due to the refraction of light rays in focusing light into samples. Therefore, in en-face imaging at each


Fig. 3 En-face OCM images of a pig thyroid gland at depths of 0.3 , $0.6,0.9,1.2,1.5$, and $1.8 \mathrm{~mm}$.

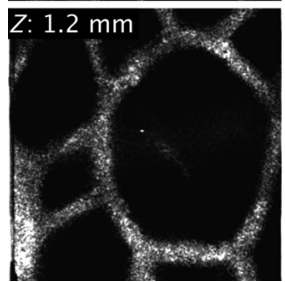

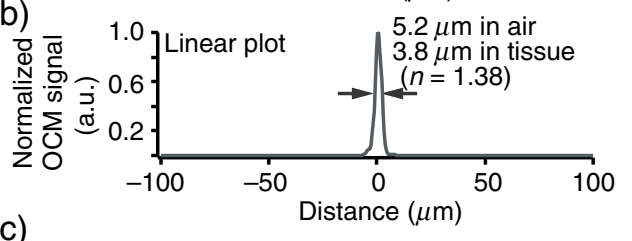
immediately after surgical removal from a pig. During the obser-

different imaging depth, the position of the reference mirror was slightly adjusted to achieve high signal intensity. The number of pixels of the images was $512 \times 512$. The image acquisition time was $6 \mathrm{~s}$ per frame. To reduce the noise, we recorded six OCM images in the same area and averaged them. In the OCM image at the depths of 0.3 and $0.6 \mathrm{~mm}$, single layers of follicular cells were clearly visualized. Although the OCM image became blurred in the observation of deeper parts of the sample due to degradation of the lateral resolution by optical aberrations induced by the sample, the characteristic follicle structures of the thyroid gland were still clearly observable. The lateral resolution could be recovered, or the degradation could be moderated, by employing adaptive optics techniques. ${ }^{23}$ The results showed that the developed 1700-nm SD-OCM has the potential to realize deep-tissue imaging with both 3-D high resolution and a large penetration depth.

In addition to the demonstration of deep tissue imaging, to investigate the merits of extending the 1700-nm OCT to OCM, we demonstrated en-face imaging of a pig thyroid gland with the 3-D high-resolution 1700-nm SD-OCM and high-axial-resolution 1700-nm SD-OCT. In this SD-OCT, by using the same SC source as that used for the SD-OCM, an axial resolution of $3.8 \mu \mathrm{m}$ and a sensitivity of $100 \mathrm{~dB}$ were achieved. The SC light was focused into samples with an achromatic lens having a focal length of $30 \mathrm{~mm}$, and the lateral resolution of the SD-OCT was $29 \mu \mathrm{m}$, which is similar to that of 1700-nm OCT reported by another group. ${ }^{20}$ Figures 4(a) and 4(b) show en-face OCM and OCT images of a pig thyroid gland at a depth of $0.3 \mathrm{~mm}$ from the surface. As shown in the result, because both OCM and OCT have the same optical sectioning capabilities, the image contrast in both OCM and OCT imaging was similar. However, whereas the OCM could resolve two layers of follicular cells separated by $11 \mu \mathrm{m}$, such structures were not observable in the OCT image, as shown in Fig. 4(b). OCM and OCT cross-sectional images at a depth of around $0.3 \mathrm{~mm}$ are also shown in Fig. 4 . Although the cross-sectional structures of a pig thyroid glands were observed with the similar axial resolution, we confirmed that the speckle size in OCM was significantly reduced by (a)

OCM (en-face)

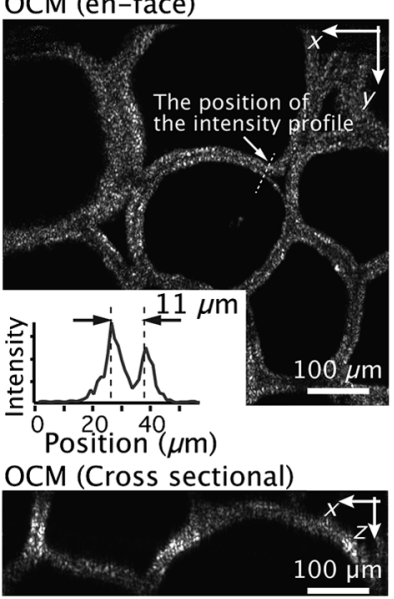

(b)

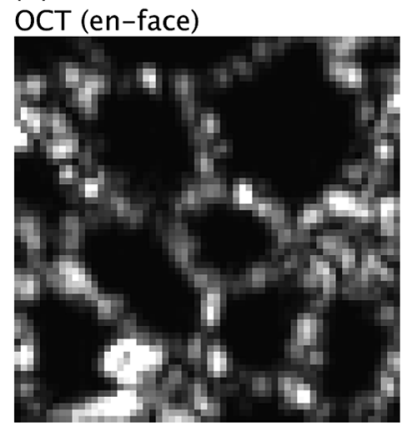

Fig. 4 En-face (a) OCM and (b) OCT images of a pig thyroid gland at a depth of $0.3 \mathrm{~mm}$. The bottom images are the OCM and OCT crosssectional images at a depth of around $0.3 \mathrm{~mm}$. The inset in (a) shows the intensity profile of the structure indicated with the white arrows in the OCM image. 
the improvement of the lateral resolution, resulting in the improvement of the visibility of sample structures. This reduction of the speckle size by improving the spatial resolution was also reported in previous OCM and OCT studies. ${ }^{24,25}$ Our demonstrations show that the extension of $1700 \mathrm{~nm}$ OCT to OCM is beneficial for biological investigations based on visualization of tissue structures in 3D.

\section{Conclusion}

In this paper, we reported the development of 1700-nm SDOCM, which enabled us to achieve both high spatial resolution in $3 \mathrm{D}$ and a large penetration depth in tissue imaging. By using an SC fiber laser source in the 1700-nm spectral band with a spectral bandwidth of $300 \mathrm{~nm}$ (FWHM) for OCM, we realized lateral and axial resolutions of 3.4 and $3.8 \mu \mathrm{m}$, respectively, with a sensitivity of $100 \mathrm{~dB}$ and an image acquisition time of $6 \mathrm{~s}$ for a single image with $512 \times 512$ pixels. With this developed SD-OCM, deep tissue imaging at a depth of up to $1.8 \mathrm{~mm}$ was also demonstrated. The adoption of adaptive optics techniques to improve the lateral resolution and numerical techniques to reduce the chromatic dispersion mismatch induced by tissue specimens would help to realize further improvements in the 3-D high-resolution imaging capability and penetration depth of the $1700-n m$ SD-OCM. ${ }^{23,26-28}$

\section{Disclosures}

The authors have no financial interests and no potential conflicts of interest.

\section{References}

1. J. A. Izzat et al., "Optical coherence microscopy in scattering media," Opt. Lett. 19, 590-592 (1994).

2. A. D. Aguirre et al., "High-resolution optical coherence microscopy for high-speed, in vivo cellular imaging," Opt. Lett. 28, 2064-2066 (2003).

3. A. D. Aguirre et al., "Cellular resolution ex vivo imaging of gastrointestinal tissues with optical coherence microscopy," J. Biomed. Opt. 15, 016025 (2010).

4. O. O. Ahsen et al., "Swept source optical coherence microscopy using a 1310 nm VCSEL light source," Opt. Express 21, 18021-18033 (2013).

5. V. J. Srinivasan et al., "Optical coherence microscopy for deep tissue imaging of the cerebral cortex with intrinsic contrast," Opt. Express 20, 2220-2239 (2012).

6. E. Beaurepaire et al., "Combined scanning optical coherence and two-photon-excited fluorescence microscopy," Opt. Lett. 24, 969-971 (1999).

7. R. Haindl et al., "Dual modality reflection mode optical coherence and photoacoustic microscopy using an akinetic sensor," Opt. Lett. 42, 4319-4322 (2017).

8. W. J. Choi and R. K. Wang, "Swept-source optical coherence tomography powered by a $1.3 \mu \mathrm{m}$ vertical cavity surface emitting laser enables 2.3-mm-deep brain imaging in mice in vivo," J. Biomed. Opt. 20, 106004 (2015).

9. U. Sharma, E. W. Chang, and S. H. Yun, "Long-wavelength optical coherence tomography at $1.7 \mu \mathrm{m}$ for enhanced imaging depth," Opt. Express 16, 19712-19723 (2008).
10. V. M. Kodack et al., "Quantitative comparison of the OCT imaging depth at $1300 \mathrm{~nm}$ and $1600 \mathrm{~nm}$," Biomed. Opt. Express 1, 176-185 (2010).

11. S. Ishida et al., "Ultrahigh-resolution optical coherence tomography in $1.7 \mu \mathrm{m}$ region with fiber laser supercontinuum in low-water-absorption samples," Appl. Phys. Express 4, 052501 (2011).

12. S. Ishida and N. Nishizawa, "Quantitative comparison of contrast and imaging depth of ultrahigh-resolution optical coherence tomography images in 800-1700 nm wavelength region," Biomed. Opt. Express 3, 282-294 (2012).

13. H. Kawagoe et al., "Development of a high power supercontinuum source in the $1.7 \mu \mathrm{m}$ wavelength region for highly penetrative ultrahigh-resolution optical coherence tomography," Biomed. Opt. Express 5, 932-943 (2014).

14. S. P. Chong et al., "Noninvasive, in vivo imaging of subcortical mouse brain regions with $1.7 \mu \mathrm{m}$ optical coherence tomography," Opt. Lett. 40, 4911-4914 (2015).

15. M. Tanaka et al., "1.7- $\mu \mathrm{m}$ spectroscopic spectral-domain optical coherence tomography for imaging lipid distribution within blood vessel," Opt. Express 23, 6645-6655 (2015).

16. H. Kawagoe et al., "Full-range ultrahigh-resolution spectral-domain optical coherence tomography in $1.7 \mu \mathrm{m}$ wavelength region for deeppenetration and high-resolution imaging of turbid tissues," Appl. Phys. Express 9, 127002 (2016).

17. M. Yamanaka, H. Kawagoe, and N. Nishizawa, "High-power supercontinuum generation using high-repetition-rate ultrashort-pulse fiber laser for ultrahigh-resolution optical coherence tomography in $1600 \mathrm{~nm}$ spectral band," Appl. Phys. Express 9, 022701 (2016).

18. H. Kawagoe, M. Yamanaka, and N. Nishizawa, "Axial resolution and signal-to-noise ratio in deep-tissue imaging with $1.7-\mu \mathrm{m}$ high-resolution optical coherence tomography with an ultrabroadband laser source," J. Biomed. Opt. 22, 085002 (2017).

19. N. Nishizawa et al., "Wavelength dependence of ultrahigh-resolution optical coherence tomography using supercontinuum for biomedical imaging," IEEE J. Sel. Top. Quantum Electron. 25, 1-15 (2019).

20. K. S. Park et al., "Deep brain optical coherence tomography angiography in mice: in vivo, noninvasive imaging of hippocampal formation," Sci. Rep. 8, 11614 (2018).

21. M. Yamanaka et al., "Optical coherence microscopy in $1700 \mathrm{~nm}$ spectral band for high-resolution label-free deep-tissue imaging," Sci. Rep. 6, 31715 (2016).

22. R. Leitgeb, C. K. Hitzenberger, and A. F. Fercher, "Performance of Fourier domain vs. time domain optical coherence tomography," Opt. Express 11, 889-894 (2003)

23. R. Fiolka, K. Si, and M. Cui, "Complex wavefront corrections for deep tissue focusing using low coherence backscattered light," Opt. Express 20, 16532-16534 (2012).

24. H.-C. Lee et al., "Ultrahigh speed spectral-domain optical coherence microscopy," Biomed. Opt. Express 4, 1236-1254 (2013)

25. P. L. Hsiung et al., "Ultrahigh-resolution and 3-dimensional optical coherence tomography ex vivo imaging of the large and small intestines," Gastrointest. Endosc. 62, 561-574 (2005)

26. A. F. Fercher et al., "Numerical dispersion compensation for partial coherence interferometry and optical coherence tomography," Opt. Express 9, 610-615 (2001).

27. M. Wojtkowski et al., "Ultrahigh-resolution, high-speed, Fourier domain optical coherence tomography and methods for dispersion compensation," Opt. Express 12, 2404-2422 (2004).

28. W. Choi et al., "Extracting and compensating dispersion mismatch in ultrahigh-resolution Fourier domain OCT imaging of the retina," Opt. Express 20, 25357-25368 (2012). 